\title{
Comunicação
}

[Communication]

\section{Ocorrência de Hepatozoon spp. (Apicomplexa, Hepatozoidae) em serpentes captive Boa constrictor mantidas em cativeiro no semiárido do estado do Rio Grande do Norte}

\author{
[Occurrence of Hepatozoon spp. (Apicomplexa, Hepatozoidae) in captive Boa constrictor snakes in \\ the semi-arid of Rio Grande do Norte State, Northeastern Brazil] \\ F.C. Lopes $^{1}$, S.S. Azevedo ${ }^{2 *}$, L.A.B. Dantas ${ }^{1}$, C.I.A. Freitas ${ }^{1}$, C.S.A. Batista ${ }^{2}$, A.S. Azevedo ${ }^{2}$ \\ ${ }^{1}$ Universidade Federal Rural do Semiárido - Mossoró, RN \\ ${ }^{2}$ Unidade Acadêmica de Medicina Veterinária - CSTR-UFCG \\ Caixa Postal 64 \\ 58700-970 - Patos, PB
}

Hepatozoon é o gênero de hemoparasitos mais comum encontrado em serpentes (Wozniak et al., 1994; Smith, 1996), e seu ciclo biológico requer dois hospedeiros: um invertebrado para a reprodução sexuada e outro vertebrado para a assexuada (Biasi et al., 1989). Os gametócitos desses protozoários, nos répteis, são observados parasitando os eritrócitos (Smith, 1996).

A identificação de espécies de Hepatozoon baseia-se principalmente na caracterização morfológica dos gametócitos em sangue periférico dos hospedeiros vertebrados e também dos cistos encontrados em órgãos internos de hospedeiros vertebrados e invertebrados (Moço et al., 2002). A descrição da ocorrência de Hepatozoon spp. em serpentes brasileiras é de fundamental importância para o conhecimento desse grupo de parasitos.

Hepatozoon juxtanuclearis (Pessoa, 1967), H. fusifex (Ball et al., 1969) e H. terzii (Sambon, 1909; Moço et al., 2002) já foram identificados em Boa constrictor, no Brasil. No semiárido do Rio Grande do Norte, não existem relatos de ocorrência desse parasito em serpentes mantidas em cativeiro. Dessa forma, o presente trabalho foi realizado com o objetivo de identificar parasitos do gênero Hepatozoon em répteis mantidos em cativeiro no semiárido do estado do Rio Grande do Norte.

Recebido em 14 de maio de 2009

Aceito em 10 de agosto de 2010

*Autor para correspondência (corresponding author)

E-mail: ssazevedo@cstr.ufcg.edu.br
Foram utilizadas amostras de sangue de Jabutipiranga, Geochelone carbonaria $(\mathrm{n}=6)$, Jabutitinga, Geochelone denticulata $(\mathrm{n}=4)$, jiboia, Boa Constrictor $(\mathrm{n}=7)$ e teju comum, Tupinanbis teguixim $(\mathrm{n}=10)$, mantidas em cativeiro no Parque Zoobotânico Onélio Porto, em Mossoró, RN. A colheita de sangue foi feita por punção da veia jugular em $G$. carbonaria e $G$. denticulata, e por punção da veia caudal em B. Constrictor e T. teguixim. Imediatamente após a colheita, procedeu-se à confecção do esfregaço sanguíneo, e lâminas foram coradas pelo método de Giemsa e analisadas em micrômetro ocular na objetiva de imersão (aumento de 1000x) e microscópio Olympus CX31. Foram observados, em média, 120 campos microscópicos por lâmina, cerca de 10.000 hemácias (O’Dwyer et al., 2004), atentando-se para a detecção da presença de gametócitos de Hepatozoon spp.

Todas as jiboias apresentaram eritrócitos infectados com Hepatozoon spp. (Fig. 1). Frequência mais baixa foi referida por O'Dwyer et al. (2003), que verificaram prevalência de $16,4 \%$ em várias espécies de serpentes estudadas. Glaser et al. (2008) analisaram serpentes Bothrops jararaca $(\mathrm{n}=10)$ e Bothrops jararacussu $(\mathrm{n}=10)$, e verificaram que $50 \%$ dos animais estavam infectados com Hepatozoon spp. Três jiboias $(42,9 \%)$ apresentaram entre $1,4 \%$ e $4,7 \%$ de eritrócitos infectados e as demais 
$(57,1 \%)$, menos de $1 \%$. Essa baixa porcentagem de parasitemia também foi encontrada por Glaser et al. (2008), que verificaram menos de $1 \%$ de parasitemia em $90 \%$ das serpentes analisadas, e por Santos et al. (2005), com variação sazonal de Hepatozoon spp. em eritrócitos de B. constrictor amarali e Hydrodynastes gigas. Biasi et al. (1972) citaram que Hepatozoon spp. pode estar presente no hospedeiro na forma latente ou com baixa porcentagem de parasitemia.

Não se sabe ao certo onde esses animais foram infectados, se no ambiente natural ou no cativeiro, no entanto sugere-se que a infecção possa ter ocorrido em consequência da ingestão de hospedeiro vertebrado intermediário infectado ou por um artrópode (carrapatos e mosquitos), aspecto já citado por O’Dwyer et al. (2003). Lainson et al. (2003), ao estudarem a esporogonia do Hepatozoon caimani em mosquitos Culex fatigans que se alimentavam em Caiman c. crocodilus e que apresentavam gametócitos em seu sangue, observaram os primeiros oocistos completamente maduros 21 dias após a alimentação dos mosquitos. Nas rãs que se alimentaram de mosquitos que continham oocistos maduros, os cistos que continham cistozoítos desenvolveram-se nos órgãos internos, principalmente no fígado. Em Caiman c. crocodilus alimentados com as rãs, apareceram gametócitos no sangue periférico desses animais. Os dados sugerem a possibilidade de ocorrer mecanismos de transmissão em condições naturais.
O presente trabalho relata a primeira descrição oficial da ocorrência de Hepatozzon spp. em $B$. constrictor em cativeiro no semiárido do Rio Grande do Norte. O estudo da ocorrência desse parasito em animais em cativeiro é de grande importância para o melhor conhecimento da saúde desses animais, uma vez que são escassos os dados a respeito dessa parasitose.

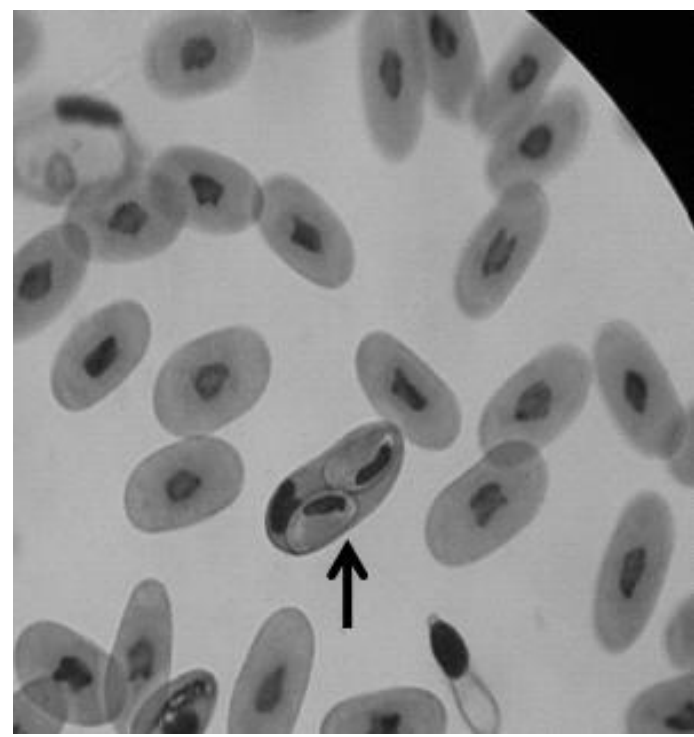

Figura 1. Gametócitos de Hepatozoon spp. (seta) em esfregaço sanguíneo de jiboia Boa constrictor.

Palavras-chave: Hepatozoon spp., jiboia, serpente, semiárido

\begin{abstract}
The occurrence of Hepatozoon spp. in the blood cells of captive Boa constritor in captivity in the semiarid of the Rio Grande do Norte State, Brazil, was analyzed. Blood samples from Geochelone carbonaria $(n=6)$, Geochelone denticulata $(n=4)$, Boa Constrictor $(n=7)$, and Tupinanbis teguixim $(n=10)$ were used. All snakes presented Hepatozoon spp. in their blood cells, and few erythrocytes were infected. G. carbonaria, G. denticulate, and T. teguixim were negative for Hepatozoon spp. gametocytes research. This is the first report of the occurrence of Hepatozoon spp. in captive B. constritor in the studied area.
\end{abstract}

Keywords: Hepatozoon spp., snake, Boa constrictor, semi-arid

\section{REFERÊNCIAS BIBLIOGRÁFICAS}

BALL, G.H.; CHAO, J.; TELFORD Jr., S.R. Hepatozoon fusifex sp. n., a hemogregarine from Boa constrictor producing marked morphological changes in infected erythrocytes. J. Parasitol., v.55, p.800-813, 1969.
BIASI, P.; PESSOA, S.P.; VIEIRA, S.C.G. Nota sobre longa latência de infecção por hemogregarina em uma serpente peçonhenta: Bothrops moojeni Hoge, 1965. Atas Soc. Biol., v.15, p.71-73, 1972. 
BIASI, P.; CARDOSO JUNIOR, R.B.; SANTOS, S.M.A. Presença de Hepatozoon plimmeri (Sambom, 1909) - Coccidia, Haemogregarinidae - em exemplar de Bothrops jararaca (Wied, 1824) - Serpentes, Viperidae, Crotalinae - mantido em cativeiro. Mem. Inst. Butantan, v.51, p.117-121, 1989.

GLASER, V.; BONI, A.P.; ALBUQUERQUE, C.A.C. Ocorrência de Hepatozoon spp. (Apicomplexa, Hepatozoidae) em serpentes do gênero Bothrops de cativeiro. Arq. Bras. Med. Vet. Zootec., v.60, p.1288-1290, 2008.

LAINSON, R.; PAPERNA, I.; NAIFF, R.D. Development of Hepatozoon caimani (Carini, 1909) Pessôa, De Biasi \& De Souza, 1972 in the caiman Caiman c. crocodilus, the frog Rana catesbeiana and the mosquito Culex fatigans. Mem. Inst. Oswaldo Cruz, v.98, p.103-113, 2003.

MOÇO, T.C.; O'DWYER, L.H.; VILELA, F.C. et al. Morphologic and morphometric analysis of Hepatozoon spp. (Apicomplexa, Hepatozoidae) of snakes. Mem. Inst. Oswaldo Cruz, v. 97, p.1169-1176, 2002.

O’DWYER, L.H.; MOÇO, T.C.; BARELLA, T.H. et al. Prevalence of Hepatozoon spp.

(Apicomplexa, Hepatozoidae) among recently captured Brazilian snakes. Arq. Bras. Med. Vet. Zootec., v. 55, p.309-314, 2003.
O’DWYER, L.H.; MOÇO, T.C.; SILVA, R.J. Description of the gamonts of a small species of Hepatozoon spp.(Apicomplexa, Hepatozoidae) found in Crotalus durissus terrificus (Serpentes, Viperidae). Parasitol. Res., v.92, p.110-112, 2004.

PESSOA, S.B. Notas sobre hemogregarinas de serpentes brasileiras. V: hemogregarinas da cascavel. Rev. Bras. Biol., v.27, p. 381-384, 1967.

SAMBON, W. The hemogregarines of snakes. J. Trop. Med. Hyg., v.12, p.48-55, 1909.

SANTOS, M.M.V.; O’DWYER, L.H.; SILVA, R.J. Seasonal variation of Hepatozoon spp. (Apicomplexa, Hepatozoidae) parasitemia from Boa constrictor amarali (Serpentes, Boidae) and Hydrodynastes gigas (Serpentes, Colubridae). Parasitol. Res., v.97, p.94-97, 2005.

SMITH, T.G. The genus Hepatozoon (Apicomplexa: Adeleina). J. Parasitol., v.82, p.565-585, 1996.

WOZNIAK, E.J.; TELFORD Jr., S.R.; McLAUGHLIN, G.L. Employment of the polimerase chain reaction in the molecular differentiation of reptilian hemogregarines and its application to preventative zoological medicine. J. Zoo Wildl. Med., v.23, p.538-547, 1994. 